
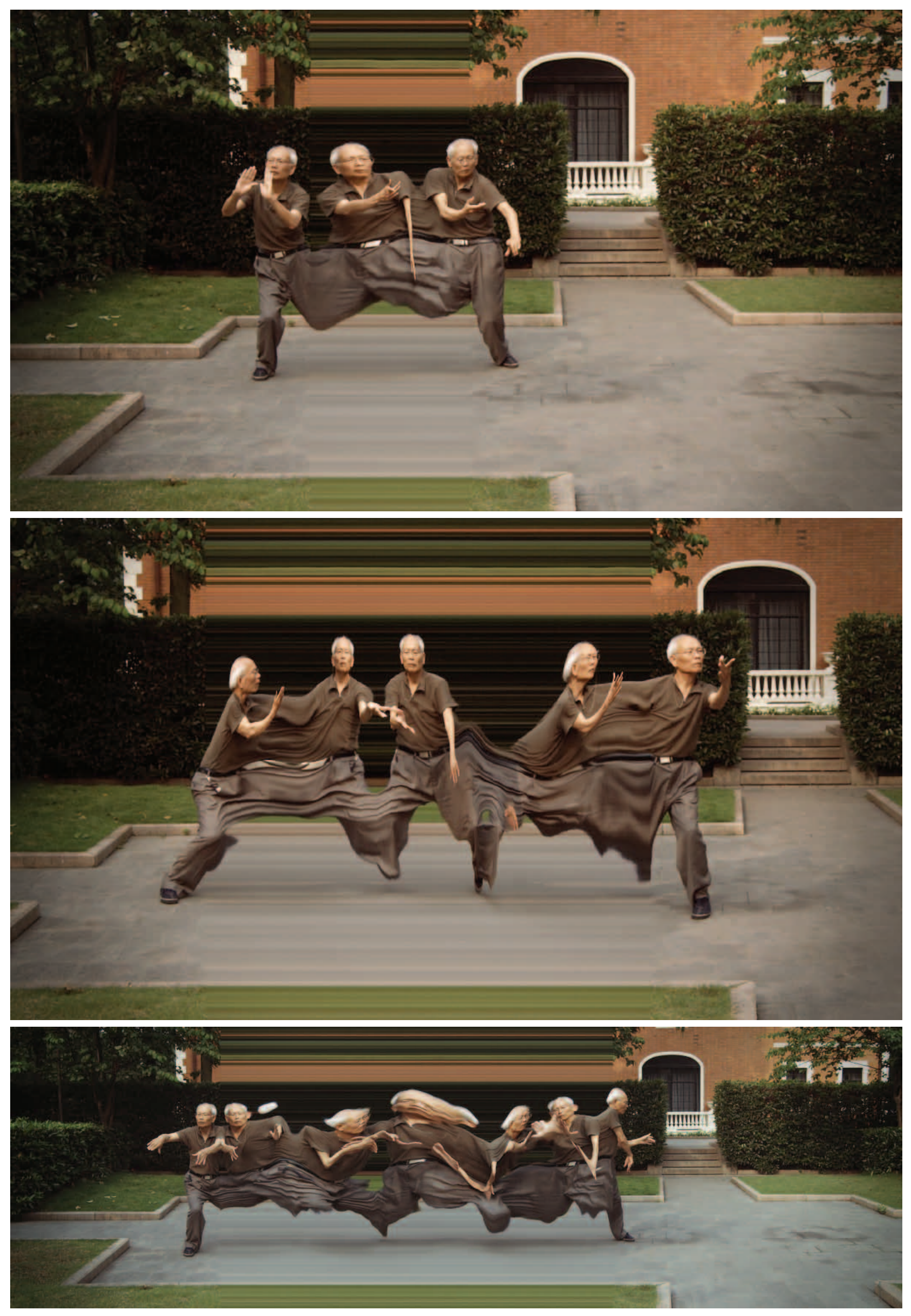


\title{
Re-Visioning Reality: Quantum Superposition in Visual Art
}

\author{
Lynden Stone
}

I

n philosophy, the usual definition of conventional reality is that it is independent, objective, meaningful and, in principle, knowable [1]; I adopt this definition for the purpose of this article. My experience of reality generally accords with this definition, insofar as I perceive objects to exist independently of me and that objects have predetermined characteristics that are independent of whatever observations or measurements I might perform on them. In particular, I can unobtrusively observe and measure objects without affecting their state or the dynamics of the system within which they exist. I sense that objects exist outside of my mind and are not just a creation of it. Naturally, I conclude that objects, other people and the rest of the physical world would exist even if I did not exist.

However, quantum mechanics [2] conflicts with the notion of a knowable, mind-independent, objective reality in a fundamental and shocking way. In contrast to the macroscopic conventional reality I perceive, in the subatomic quantum world, there is no physical matter as I understand it. Rather, according to the accepted formulation of quantum mechanics, states of pre-matter exist in a superposition consisting of a connected wave/particle duality ("wavefunction") of multiple possibilities that, according to conventional views, is collapsed and brought into a definite state of singular material reality through my own observation or measurement [3]. Rather than reality being knowable, objective and mind-independent, conventional quantum theories pose the confounding possibility that there is a quantum realm imperceptible to and unknowable by me and that the reality I experience is brought into existence through my measurements or observations [4]. In a further challenge to my perception of reality, quantum particles can exist in states of "entangled" superposition that indicate an inexplicable interconnectivity of physical matter.

The features of quantum mechanics and their contradiction to conventional reality are the basis for the persistent call for a reevaluation of our notion of conventional reality. In 1935, Niels Bohr called for a "radical revision of our attitude towards the problem of physical reality" [5]. Werner Heisenberg noted that the change to the concept of reality proposed by quantum theory was not a continuation of past concepts; rather, "it seems to be a real break in the structure of modern science" [6]; David Deutsch suggests that quantum theory "require[s]

Lynden Stone (visual artist, scholar), Queensland College of Art, Griffith University, Brisbane, Australia; 35 Deerhurst Road, Brookfield, Queensland, Australia, 4069. E-mail: <lyndenstone@gmail.com>; URL: <www.lyndenstone.com.au>.

See $<$ www.mitpressjournals.org/toc/leon/46/5> for supplemental files associated with this issue.

Article Frontispiece. Daniel Crooks, Static No. 12 (seek stillness in movement) (details of video stills) HD digital video 05:23 min, 16:9, 1080p24, stereo, 2010. () Daniel Crooks. Image courtesy of the artist and Anna Schwartz Gallery.) a radical departure from the classical conception of the fabric of reality" [7], and Karen Barad states that we need a "reassessment of physical and metaphysical notions that explicitly or implicitly rely on old ideas about the physical world" [8]. Trying to understand a world described by quantum theory, says philosopher Lawrence Sklar, requires a far more radical revision of our understanding of the nature of things than that demanded by the theories of relativity [9].

Quantum mechanics is mindboggling, counterintuitive and tricky to grasp. A famous quote is attributed to Richard Feynman: "If you think you understand quantum mechanics, you don't understand it." Feynman, however, did definitely say, "I think I can safely say nobody understands quantum mechanics" [10]. For nonscientists, the mathematical formalism used to explain quantum concepts acts as a further barrier to understanding. Visual art, however, can provide ways of conceiving these concepts outside the bounds of scientific formalism, possibly facilitating the necessary conceptual revision. Physicist and philosopher David Bohm believed that art was a vehicle capable of exploring "fundamentally new modes of perception, through the senses and new forms of imagination" [11]. Because visual art relies on methods such as metaphor and the phenomenal, it can arguably represent quantum concepts in a more comprehensible form than can the mathematical formula. Barbara Stafford suggests that images can simplify a complex environment conceptually and also make "aspects of the world perceptually salient and cognitively distinctive for us" [12].

Representing quantum concepts through visual art, however, is problematic and challenging. Although quantum effects are puzzling, uncertain and inchoate, they are necessarily described using the concepts of classical physics, which is precise and bears relation to our physical world. The available language of description limits what can be described. The artist, too, is limited by the language and connotation of the materials used to convey the immaterial. Metaphors and symbols from our experience of reality may be inadequate, imprecise or powerless to suggest the imperceptible. Furthermore, due to the limiting factors of materiality and interpretation, any representation of quantum concepts may be criticized as erroneous. These factors limit the effectiveness of visual representations of quantum concepts. In the second part of this paper, in focusing on quantum superposition, a fundamental mystery of quantum mechanics, I examine how visual representations by artists Jonathon Keats, Julian Voss-Andreae, Antony 
Gormley and Daniel Crooks address these challenges and how their work might provide insights into quantum superposition that, in turn, might help us revise our perception of reality.

\section{JONATHON KEATS: QUANTUM ENTANGLEMENTS}

Conceptual artist Jonathon Keats invites participants to contemplate the bizarre properties of quantum entanglement (i.e. generalized superposition involving more than one particle [13]) through interaction with his Quantum Entanglements (2011) (Fig. 1). In the work, a particle beam splitter (Fig. 2) was installed in a New York gallery in 2011 [14]. According to Keats, a nonlinear crystal beam splitter uses sunlight to spray entangled pairs of photons onto couples standing at designated spots on the gallery floor. Physicists can create such entangled pairs of photons by sending them through crystal beam splitters. Separated parts of the same entangled system have been observed to remain in a connected state of superposition. A measurement or observation on one of the separated parts influences the other instantaneously (faster than the speed of light), even when they are separated by vast distance. This conflicts with our experience of local realism (i.e. that only objects that share a proximate relationship to each other are perceived to affect each other). In quantum theory, the fact that quantum objects that do not share proximity can be affected by each other suggests a possible connectedness or universality that is not observed as part of our everyday reality, at least suggesting that "particles that were once together in an interaction remain in some sense parts of a single system, which responds together to further interactions" [15]. A measurement made on one half of the separated entangled pair that instantly affects the separated half also conflicts with our experience that an observation or measurement on an object does not affect the state or system dynamics of the object.

Keats purports to foster human relationships through the use of his device, offering his form of union and "entanglement" as an alternative to the manmade laws of marriage. The "entanglement" effect of the artwork (supposedly to bind couples together even though they may be separated by distance), he says, is a matter to be taken on faith, as any attempt to measure the entanglement will disentangle it [16]. Keats is referring to the capacity of quantum systems to remain in a state of non-
Fig. 1. Jonathon Keats, Quantum Entanglements, 2011, installation shot.

(๔ Jonathon Keats)

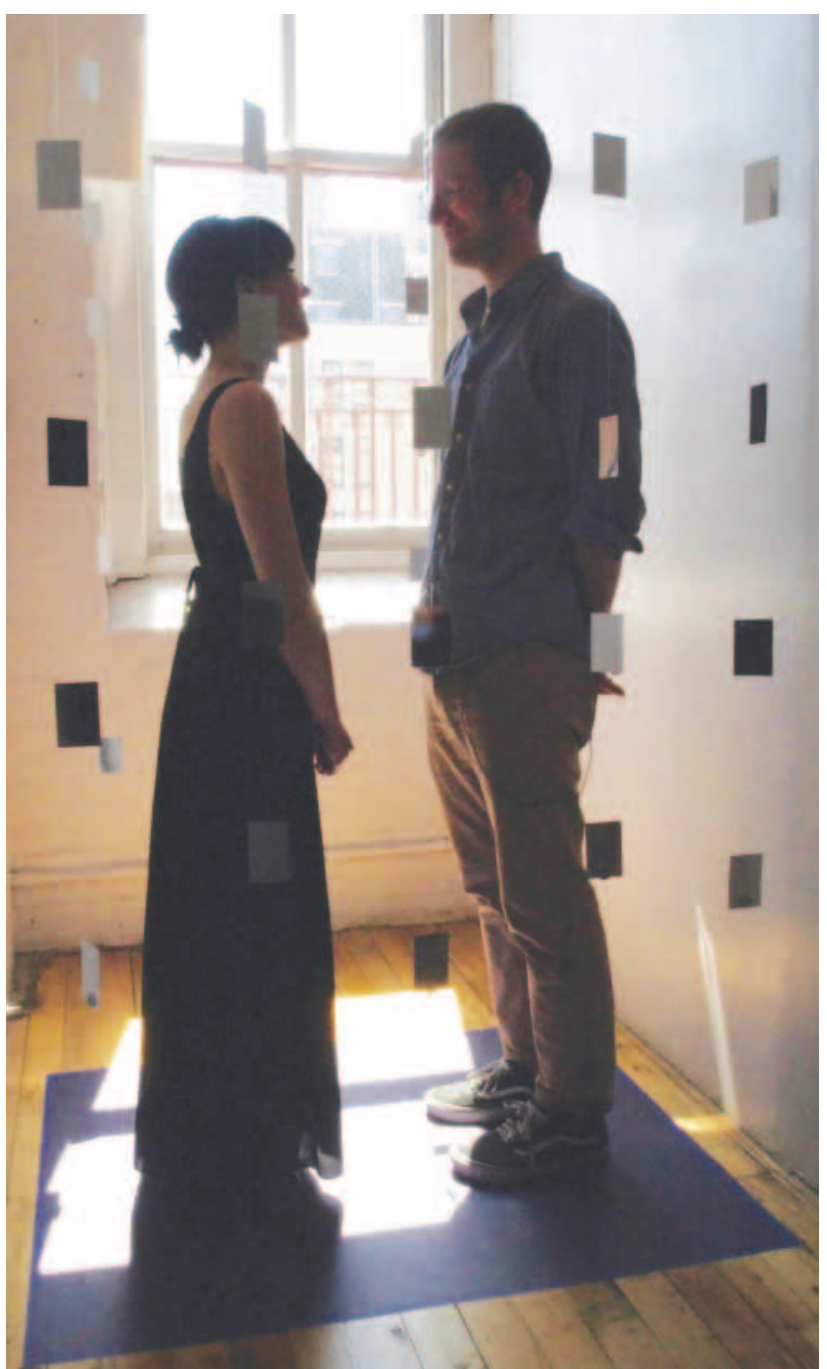

definite superposition until measured or observed.

Keats addressed the paradoxical nature of describing non-material quantum superposition in the material world by using little in the way of materiality for Quantum Entanglements: a lens, a crystal and a mirror secured to a small metal base plate to catch the sun, a mat on the floor, some dangling mirror pieces-the latter seemingly filling only aesthetic function. This minimal materiality of the work is in stark contrast to the expansive and confounding ideas behind superposition and entanglement.

Apart from the sunlight they stand in, couples that interact with Keats's Quantum Entanglements presumably discern no particular physical effects and cannot be sure that they are being sprayed with entangled photons. Keats, however, seems confident that his device produces entangled photons. While he professes a concern with "baseline scientific validity" to provide a solid springboard and give traction to his metaphor, his interest is not whether permanence of entanglement persists but rather that en- tanglement "between two people-at an atomic level-is likely to take place as a result of interaction with my apparatus" [17].

Physicist Geoff Pryde says that Quantum Entanglement would fail to effect any real quantum entanglement [18]. Pryde is Associate Professor in the Centre for Quantum Dynamics at Griffith University and conducts research into the application of entangled quantum systems for computing and encryption. He observes in response to Keats's work that, while absorption of the photons by atoms in the bodies of couples that have been sprayed with entangled photon pairs may briefly entangle those atoms, the interaction of those atoms with the myriad other atoms in their bodies or in their environment will result in either a measurement collapsing the superposition and entanglement or a washing out of the information carried by the particles. In his view, then, Keats's Quantum Entanglements is unlikely to result in any lasting "entanglement" of parts between two people. However, Pryde places two qualifications on his opinion. The first is that a single 
photon could interact with a single atom in each person's body, thereby forming a quantum system in superposition. However, he says, the atoms in each person are so strongly coupled with the rest of the systems in their bodies that any quantum system would likely collapse, ending any entanglement. Secondly, his opinion is based on the theory of environmental collapse of quantum superposition [19] not observation or measurement by human intervention. There is a small minority of quantum theorists [20] who believe, unlike Pryde, that it is only the conscious mind of an observer that can collapse quantum superposition states; if this theory is correct, an entangled superposition state could persist in the absence of any deliberate observation or measurement of absorbed photons sprayed by Jonathon Keats's beam splitter.

Quantum Entanglements raises the issue of the extent to which artists who make work referencing scientific concepts should do so accurately. Physicists have objected to the inappropriate extrapolation of quantum mechanics as the scientific foundation for para-scientific, supernatural and spiritual concepts [21]. Should creative interpretation and expression of quantum concepts be stymied by the insistence on their conformity with scientific accuracy? Visual art is a creature altogether differ-

Fig. 2. Jonathon Keats, Quantum Entanglements, detail of particle beam splitter. (๔ Jonathon Keats)

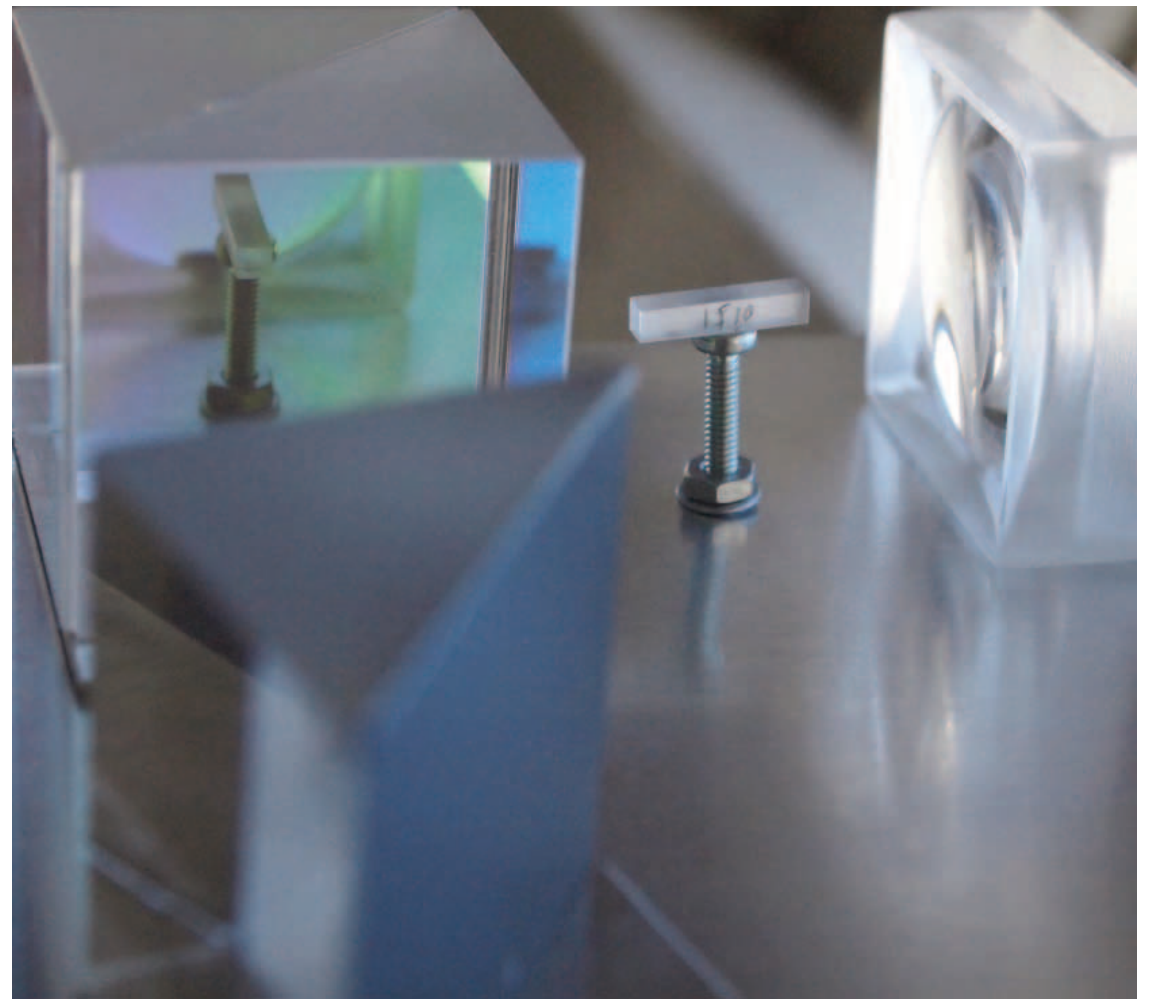

ity does exist, and it has real application in the macroscopic world in the field of quantum encryption.

\section{JULIAN VOSS-ANDREAE}

In December 2010 I visited sculptor and physicist Julian Voss-Andreae in his Portland, Oregon, studio. Listening to him instruct fellow artist and master welder Jordan Tull on the assembly of another edition of Voss-Andreae's metal sculpture Quantum Man (2007) (Fig. $3)$, I was struck by the materiality surrounding me. Metal sheets and bars, overhead gantries-here was the stuff of classical physics, being used to make works representing the non-material. The contradiction struck me as both ironic and frustrating, but one that most artists working with these concepts face. Werner Heisenberg expressed a similar frustration when he observed that, when describing quantum experiments, we are limited by the applicability of classical concepts to what is being described. Nevertheless, he said, we cannot and should not try to improve them [22]. In making art about quantum concepts, we must realize that the materials we use can never properly conjure the immaterial uncertainty of the quantum world.

Voss-Andreae has made a body of work called Quantum Sculptures about different quantum concepts; these works are the subject of a previous article in Leonardo [23].

In 1999 , as a postgraduate physicist with Anton Zeilinger's experimental physics group in Vienna, Voss-Andreae was part of a team that used buckminsterfullerene molecules in a quantum double slit experiment to show an interference pattern, indicating that, in addition to sub-atomic quanta, microscopically visible particles also demonstrate the state of wave/particle duality characteristic of quantum superposition. Recently, however, physicists Kofler and Brukner demonstrated that quantum superposition is observable in increasingly larger macroscopic objects, leading them to support a non-collapse theory: that the wavefunction of the quantum world may never break down. They propose that the classical world is made up of quantum states of superposition that are simply undetectable by the coarse measuring devices of the macroscopic world [24]. Quantum Man is an exploration of quantum superposition, of what we might be able to see if our eyes were not such coarse measuring devices. VossAndreae says the sculpture is a visualization of what the quantum wavefunction of a walking person might look like [25]. 

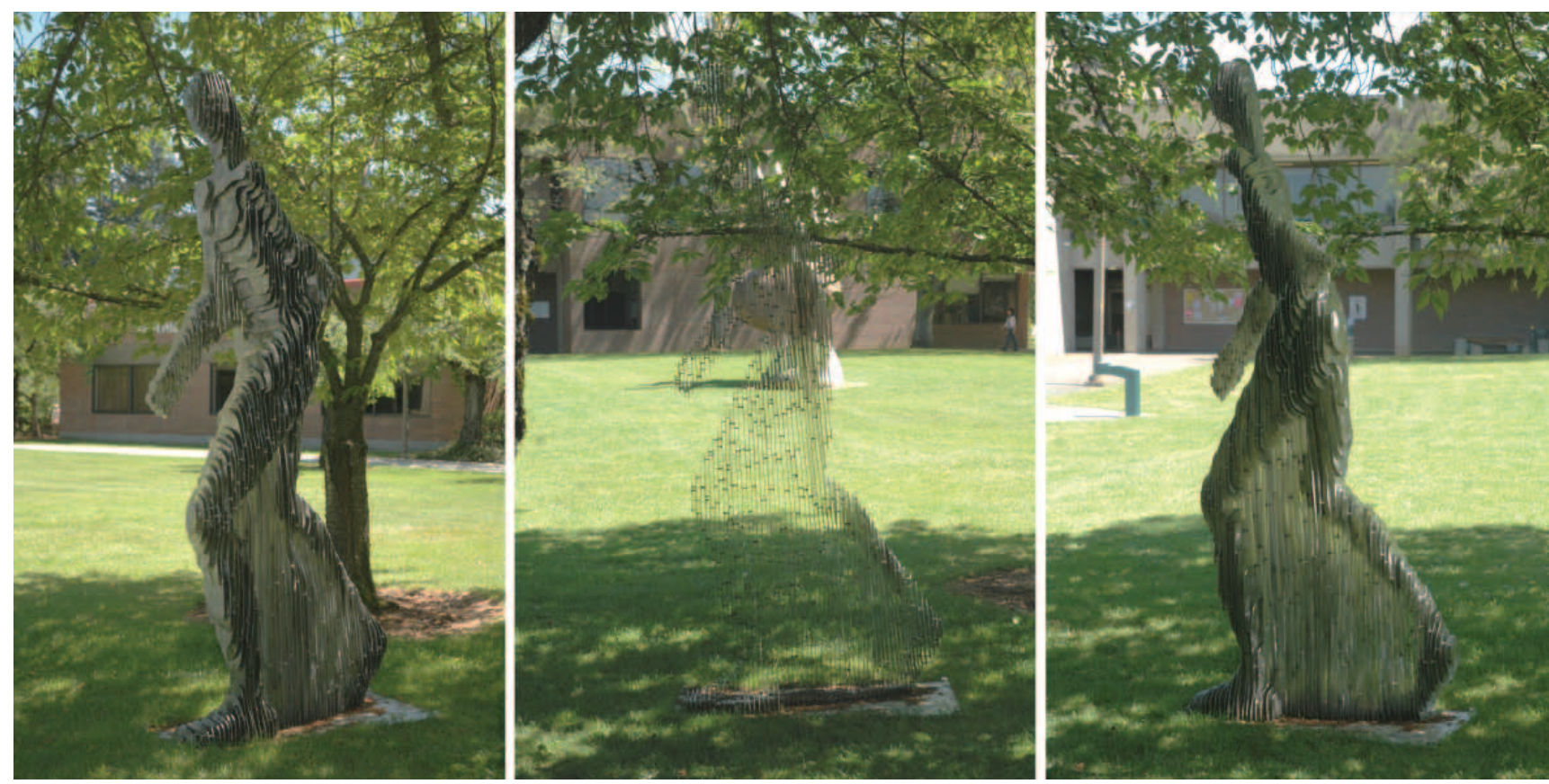

Fig. 3. Julian Voss-Andreae, Quantum Man, stainless steel, $250 \times 110 \times 50 \mathrm{~cm}, 2007$. Photographed at the Clackamas Community College "Sculpture Exposition," 2007, Oregon. (๔ Julian Voss-Andreae)

This larger-than-life sculpture is made of thin, welded sections of metal. When Quantum Man is viewed front-on, the metal form appears to fill out and occupy the space almost as one solid form. However, viewed from the side, the figure wondrously all but disappears, leaving only fine slivers suggesting a human figure. Thus, surprisingly, the same object appears solid and dense from one angle and fine and insignificant from another.

Voss-Andreae's Night Path (2009) (Fig. 4) is a representation of physicist Richard Feynman's "path integral" calculations in quantum mechanics. Feynman calculated the probabilities that a single particle could travel along all possible paths between two points at set intervals of time. These paths, however, do not exist. They exist only as mathematical expressions of the superposition of all possible states. According to quantum collapse theories, when the particle is observed, only one path is taken by the particle. The "paths" in Voss-Andreae's sculpture are fine gold threads set between 10 slices of thin steel. This work is a material representation of an event that cannot exist within our perception.

Does this piece, like Niels Bohr's outdated model of the atom, give us an inaccurate, proscriptive representation of superposition states, or does it help in explaining the weird concept of quantum superposition? Bohr's model, developed early in the 20th century, prior to the understanding of quantum physics, is a legacy that is culturally embedded but completely false. Bohr himself knew that, despite his model, an atom is nothing like a miniature solar system, with electrons whizzing around a nucleus in perfect orbits. This inaccurate representation persists, however [26]. Unlike the Bohr model, Voss-Andreae's Night Path is based on the diagrammatical mathematics of quantum mechanics. It is a representation of what mathematics holds to take place in a state of quantum superposition, which adds some veracity to the work. Historian and philosopher of science Arthur Miller points out that the visual imagery of pre-quantum subatomic theories was inaccurate and confusing, but the imagery of quantum mechanics represented by Feynman's diagrams "is generated by the theory's mathematics" [27]. While Night Path is closely based on Feynman's diagrams, its careful threedimensional construction and gold thread and scale help to take it beyond the status of a mathematical model.

Perhaps due to his scientific training, Voss-Andreae's works translate scientific quantum concepts into the physical without seeking extrapolation into disputed quantum theories. Arguably, his works prompt fewer (if any) objections of scientific inaccuracy by physicists than does

Fig. 4. Julian Voss-Andreae, Night Path (detail), painted steel, golden thread, $46 \times 48 \times 15$ cm, 2009. (@ Julian Voss-Andreae. Photo: Dan Kvitka.)

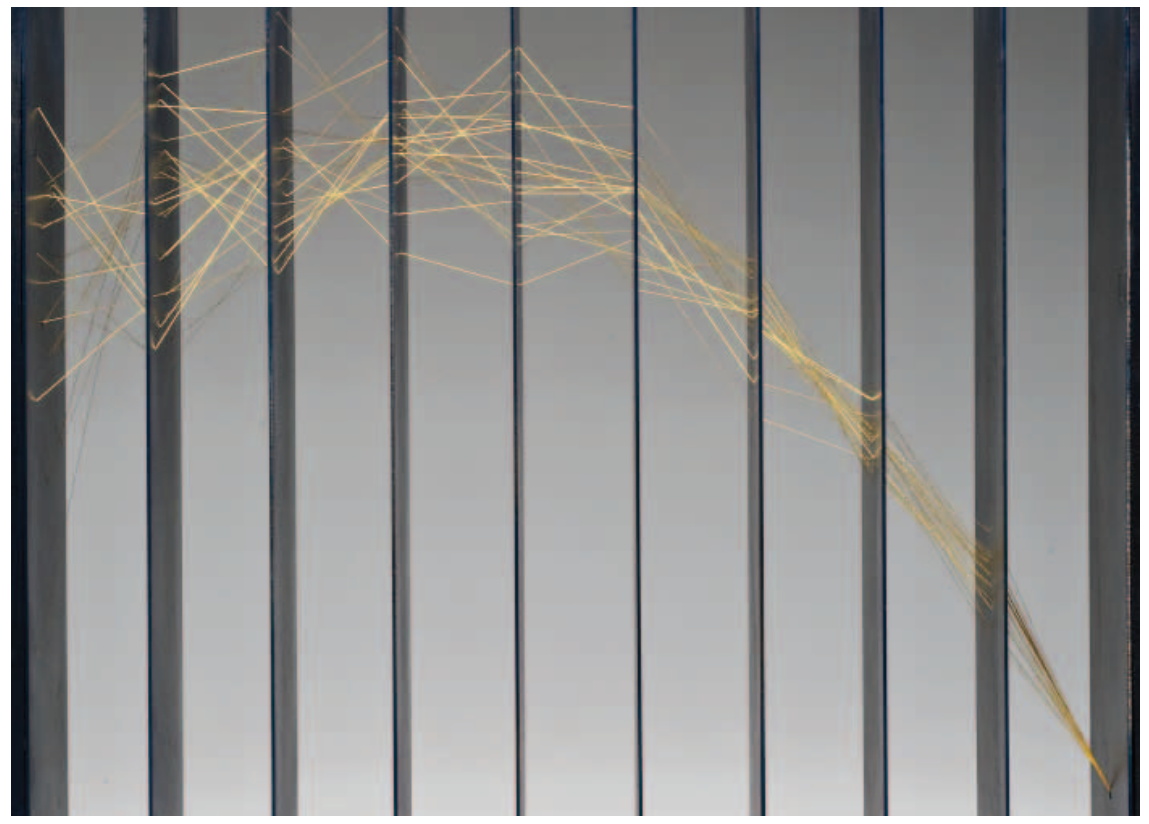


a work such as Keats's Quantum Entanglements. Voss-Andreae is aware, however, that his artwork cannot be held up as definitive, objective representation of quantum concepts, as quantum physics, he says, does not support objective reality [28].

Relying on the phenomenology of most of the objects he makes, VossAndreae does not attempt to shy away from materiality. Materiality, however, contradicts the principle of indeterminacy of quantum superposition. Nevertheless, these objects can hint at the unknown reality beyond their physical structure. Quantum Man and Night Path are material imaginings of a state that is ordinarily beyond our perception. They represent two separate theories of superposition: one, in which superposition in our physical world does not collapse, and a corollary theory, where all possible positions collapse into one outcome. Perhaps, of the two works, Quantum Man, because it resembles the human form, is more successful in conveying the idea that wave/particle superposition is part of our extended reality.

\section{ANTONY GORMLEY}

Gormley's series of Quantum Cloud sculptures [29] investigates wave/particle duality and the possibly subjective nature of quantum reality. Part of that series, Quantum Cloud (1999) (Fig. 5), was commissioned for a site over the Thames River beside the Millennium Dome in London. It is 30 meters high and constructed of 1.5-m-long sections of steel. During construction of the work, Gormley was reported as saying (presumably with his tongue firmly planted in his cheek) that, as the work was based on quantum uncertainty, he did not know whether a body would be discernible in the cloud. "The problem is," he said, "that you can't have your cake and eat it: you can't have quantum reality and recognise a body" [30]. Although amusing, this point underlies what might have been a frustrating truth for Gormley: that, as with Bohr's model of the atom, one cannot have an objective physical representation of the superposition of wave/particle duality or of any quantum reality. The physical representation must always be metaphorical.

Of course, classical materiality prevailed, and a figure can be discerned inside Gormley's Quantum Cloud. However, it is the scale of Quantum Cloud that conveys a sense that material things may not be as "material" as we may think. In the recent BBC production "The Story of Science," presenter Michael Mosely
Fig. 5. Antony

Gormley, Quantum

Cloud, galvanized steel,

$29 \times 16 \times 10 \mathrm{~m}, 2000$.

(C) Antony Gormley.

Photo: Andy Roberts.

Image source:

Wikipedia,

<www.wikipedia.org>.)

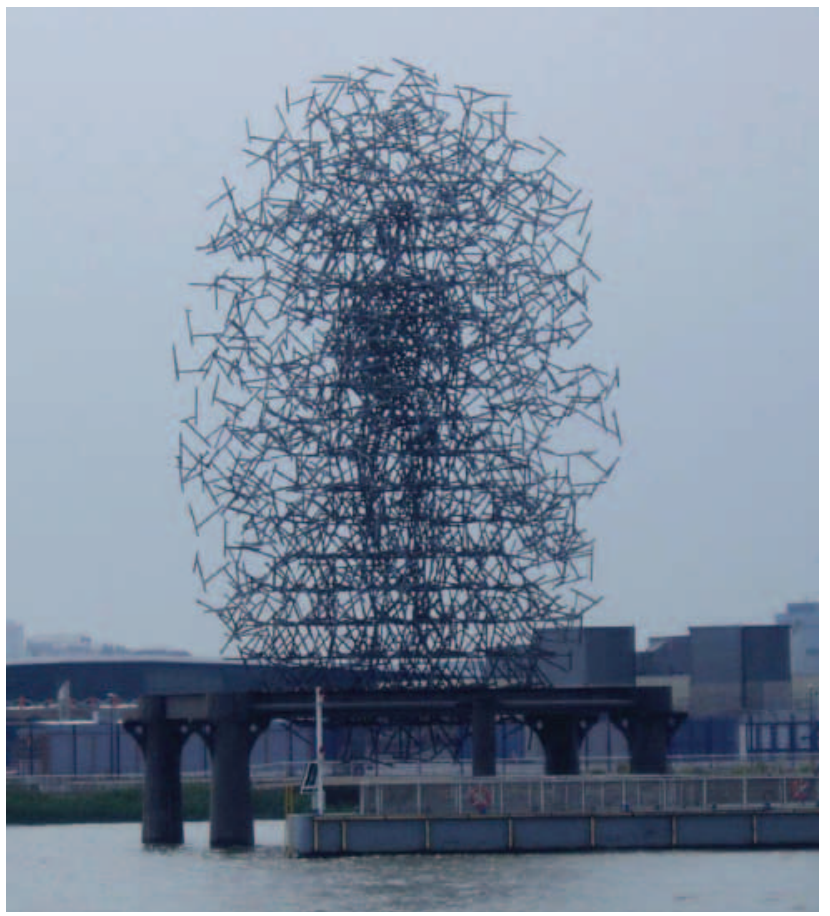

claimed that, if you took all the space out of all the people on earth, the remaining material would be the size of a sugar cube [31]. This minimal materiality of physical reality is suggested in Quantum Cloud; the separated parts emphasize vacant areas of space.

Quantum Cloud achieves a mixture of inchoateness and meaning. Like a physicist making a quantum measurement, by gazing up at the Quantum Cloud I become the measuring device, the agent acting upon the system and causing the wave/particle duality to "collapse" into material reality. I construct the meaning of the work through my subjective, phenomenological response of seeing the figure. The process is evocative of the quantum concept of non-objective reality; when I look away, does material reality re-assume its superposition because I am not looking? In classical physics, we assume that physical systems have pre-existing material characteristics independent of other systems that can be ascertained by observation and measurement. In the quantum world, however, there is no predetermined definite state. Contrary to our usual experience of conventional realism, it is only the interaction with a measuring device that yields definite, physical results [32].

Einstein and his collaborators held that quantum physics must be incomplete, because "no reasonable definition of reality" could be based on whether or not something is observed or measured [33]. However, quantum physics is based on this definition of reality. In quantum physics, the process of observation or measurement disturbs superposition to disclose the material particle [34]. More recent theories account for the collapse of the wave/particle state by exposure to and entanglement with the environment. On any view, the problem of when and how the state of superposition of wave/ particle duality is collapsed into particle reality is troubling and intriguing. With Quantum Cloud, Gormley seems to have found a way to express the baffling concept that something only becomes "real" when measured or even merely observed by us.

\section{DANiel Crooks}

When I first saw Daniel Crooks's digital video Static No. 12 (seek stillness in movement) (Article Frontispiece), at the 2010 Sydney Biennale, I instantly drew comparisons to the quantum superposition state, in which quanta exist in a wave/ particle duality of all possibilities. I spoke to Crooks at the Biennale, and although he does not concede that his work directly references quantum concepts, the comparison is hard to ignore. Crooks admits an interest in time and space, but it seems that he has hit upon metaphors for quantum superposition unconsciously. In his artist's statement for a similarly constructed work, he wrote that he endeavors "to trigger a perceptual shift in our viewing of the space/ time continuum, graphically revealing the underlying rhythms and patterns of the physical world and tracing rhythms of our navigation through it" [35].

In Static No. 12 (seek stillness in movement), Crooks decelerates time by taking 
slivers of video sequences and expanding them across the entire frame. In this way, figures stretch through space and time, persisting at points in space longer than we would normally perceive. They exhibit a fluid, elastic motion, expanding and contracting, like the visual "trails" of an LSD experience [36].

Working out where the quantum world of superposition ends and the material macroscopic world we perceive begins continues to confound physicists. However, Kofler and Brukner's observation of quantum effects in increasingly larger macroscopic objects leads them to propose that the classical world is made up of quantum states of superposition undetectable by coarse measuring devices in the macroscopic world [37]. In Static No. 12, a man moves languidly, as if walking through viscous gel. However, as he moves through space, it is the man's form, and not the invisible substance through which he moves, that is impacted by the movement. The trails of his image suggest the persistence of quantum superposition states in which constituent parts are spread out-indeterminate but coherently linked.

Through the nature of the digital moving image, and without the heaviness of physical materials, Crooks is able to provide a compelling visual rendering of the humanly imperceptible reality of a state of superposition. The changing but wholly connected image conjures inter-related, flowing states in which possibilities for movement exist throughout. Even though the content-rich Static No. 12 implies hours of intensive editing, the viewer, in a darkened room with light streaming conically from the projector onto a simple white flat screen, has the impression of a lack of real materiality to the work. Compared to the static, material nature of Voss-Andreae's Quantum Man and Gormley's Quantum Cloud, Crooks's video has a mesmerizing and captivating effect, allowing a viewer to stay with and contemplate the work.

\section{CONCLUSION}

If we think of reality as a mind-independent, objective and knowable concept, then features of the quantum world challenge that reality. Physicists have called for a reevaluation of our view of reality to incorporate quantum features, and visual art can assist this re-evaluation. Although problematic and paradoxical, in that material and metaphors from our reality must be used in varying degrees, the artworks of Jonathan Keats, Julian Voss-Andreae, Antony Gormley and Daniel Crooks all, to some extent, provide a macroscopic translation of superposition-a fundamental mystery of quantum physics. In some ways these artworks help to expand our awareness that reality may be more than just the seemingly objective, mind-independent world that we perceive with our senses. They may also provoke a radical departure from or revision of our views of conventional reality. At the very least, they introduce quantum concepts to a wider audience through translations freed from the mathematical formalism of quantum physics. These works are a step toward grasping the bizarre reality of the quantum world.

\section{Acknowledgments}

I wish to acknowledge the assistance of Geoff Pryde for his comments and clarifications on matters of quantum entanglement. I acknowledge the helpful notes of Richard Dunn and of other anonymous reviewers. Thanks also to Mostyn Bramley-Moore and George Petelin for their comments, suggestions and continued support.

\section{References and Notes}

\section{Unedited references as provided by the author.}

1. B. d'Espagnat, "Quantum Physics and Reality", Foundations of Physics, Vol. 41, Issue 11, 1703-1716 (2011) pp. 1712-1713.

2. Quantum mechanics is the mathematical description of the world at the smallest, sub-atomic scale. In this article, I use the term quantum physics in a general, overarching sense that includes quantum mechanics and quantum theory. I use quantum mechanics to mean the mathematical formulation of quantum physics and quantum theory to refer to the conceptual basis of quantum physics and quantum mechanics.

3. W. Heisenberg, Physics and Philosophy (New York: Harper Torchbook [1958] 1962) pp. 54-55.

4. See F. London and E. Bauer, "The Theory of Observation in Quantum Mechanics", (1939) in Quantum Theory and Measurement, J.A. Wheeler and W.H. Zurek (eds.) (New Jersey: Princeton University Press 1983), pp. 217-259: p. 251; J. Wheeler, "Law Without Law", in Quantum Theory and Measurement, J.A. Wheeler and W.H. Zurek (eds.) (New Jersey: Princeton University Press 1983) pp. 182-213: pp. 184-185; E.H. Walker, The Physics of Consciousness (Cambridge: Perseus Publishing, 2000); B. Rosenblum and F. Kuttner, "The Observer in the Quantum Experiment”, Foundations of Physics, Vol. 32, No. 8, 1273-1293 (2002).

5. N. Bohr, "Quantum and Physical Reality," Physical Review, Vol. 48, 696-702 (1935) p. 697.

6. Heisenberg [3] p. 29

7. D. Deutsch, The Fabric of Reality (New York: Penguin Books, 1997) p. 327.

8. K. Barad, Meeting the Universe Halfway: Quantum Physics and the Entanglement of Matter and Meaning (Durham NC: Duke University Press, 2007) p. 24.

9. L. Sklar, Philosophy of Physics (Boulder, Colorado: Westview Press, 1992) p. 157.

10. R. Feynman, "Probability and Uncertainty: The Quantum Mechanical View of Nature," Lecture 6 of The Character of Physical Law series, Cornell University, <www.youtube.com/watch?v=kekayfI8Ii8>, (1964).

11. D. Bohm, On Creativity (London: Routledge, 1996) p. 134.
12. B. Stafford, Echo Objects (Chicago: University of Chicago Press, 2007) p. 207.

13. Barad [8] p. 270.

14. 12 May-18 June 2011, AC Institute Gallery, <www. artcurrents.org $>$.

15. J. Gribbin, In Search of Schrodinger's Cat (London: Black Swan, 1984) p. 229.

16. AC Institute, "Jonathon Keats: QUANTUM ENTANGLEMENTS," <www.artcurrents.org> (accessed 7 May 2011).

17. J. Keats, email to the author (23 June 2011).

18. G. Pryde, in discussion with the author (15 May 2011).

19. Pryde [18].

20. For instance, E. Wigner, Symmetries and reflections (Bloomington: Indiana University Press, 1967) p. 171-184; David Bohm, "A new theory of the relationship of mind and matter," Philosophy Psychology, Vol. 3, No. 2, 271-286 (1990); Henry Stapp, "Quantum Interactive Dualism: An Alternative to Materialism," Zygon, Vol. 41, No. 3, 599-615 (2006).

21. For instance see V. Stenger, "The Myth of Quantum Consciousness," The Humanist, Vol. 53, No. 3, 13-15 (1992)

22. Heisenberg [3] p. 44.

23. J. Voss-Andreae, "Quantum Sculpture: Art Inspired by the Deeper Nature of Reality," Leonardo, Vol. 44, No. 1, 14-20 (2011).

24. J. Kofler and C. Brukner, "Classical World Rising out of Quantum Physics under the Restrictions of Coarse-Grained Measurements," Physical Review Letters Vol. 99, 180403(4) (2007) p. 1.

25. Voss-Andreae [23] p. 15.

26. Gribbin [15] p. 53.

27. A. Miller, Einstein, Picasso-Space, Time and the Beauty That Causes Havoc (New York: Basic Books, 2001) p. 256.

28. J. Voss-Andreae [23] p. 17.

29. <www.antonygormley.com/sculpture/series $>$.

30. New York Times, "Antony Gormley-Diary" (3 November 1999).

31. The Story of Science, "What is the World Made of?" London, BBC television documentary (2011).

32. Heisenberg [3] pp. 54-55.

33. A. Einstein, B. Podolsky and N. Rosen, "Can Quantum-Mechanical Description of Physical Reality Be Considered Complete?" Physical Review, Vol. $47,777-780$ (1935) p. 780.

34. Heisenberg [3] pp. 54-55.

35. D. Crooks, Artist Statement, National Gallery of Australia (2006), <www.nga.gov.au/fullscreen/06/ crooks.pdf $>$.

36. J. Dubois and R. Van Rullen, "Visual trails: Do the Doors of Perception Open Periodically?" PLOS Biology, Vol. 9 Issue 5, e1001056, 1-4 (2011).

37. Kofler and Brukner [24].

Manuscript received 14 November 2011.

Lynden Stone is a visual artist and Ph.D. candidate in Fine Art at the Queensland College of Art, Griffith University. Her research project is to explore, through her studio practice and that of others, how visual artists can represent the enigmas that the quantum world presents to our perceived reality. She lives in Brisbane, Australia. 


\section{A WORD OF THANKS}

\section{Thanks to Our Reviewers}

The Leonardo publications are peer reviewed: before acceptance, every article is reviewed and commented on, usually by three anonymous reviewers. Based on these reviews we make a decision whether to publish the text.

Leonardo covers a wide variety of fields, many of them interdisciplinary and often in emerging areas of experimentation, research and scholarship. Our peer reviewers help us focus on new topics of interest, as other areas may move out of the center of experimentation and become part of established disciplines. In very new areas of inquiry it is often difficult for us to identify three reviewers competent to review the work; in these cases we may ask the authors themselves to suggest new reviewers for our peer review panel. The Leonardo Network in a very real sense grows and evolves through this process of collaborative filtering.

We wish to take this opportunity to thank some of the reviewers who in the past year have on a volunteer basis spent time writing very thoughtful reviews that both help us make a publication decision and help the authors to improve their texts:

Liliana Albertazzi, Christine Assaiante, Marc Battier, Sinan Bokesoy, Claus-Christian Carbon, David Carrier, Michael Corballis, Mellisa Coleman, Evelina Domnitch, Frank Dufour, Michael Early, David Em, Michele Emmer, Jayanne English, James Enns, Kathryn Evans, Sidney Fels, Jessica Field, Ken Fields, Michael Fowler, Jean Gagnon, Scot Gresham-Lancaster, Amanda Gluibizzi, Brian Harnetty, Amy Ione, Susan Iverson, Janis Jefferies, Mauri Kaipainen, Jeremy Kargon, Kim Knight, Francois-Joseph Lapointe, Patrice Le Gal, Guillermo Lemarchand, Jerrold Levinson, Patrick Lichty, John Mallinckrodt, Aleksandra Manczak, Edgar Meyer, Rosemary Mountain, Frieder Nake, Michael Neff, Stephan Neuhauss, Michael O'Shea, Jin Wan Park, Sidney Perkowitz, Lucy Petrovic, Cliff Pickover, Sheila Pinkel, Patricia Pisters, Vincent Racaniello, Keith Rayner, Joana Ricou, Robert Root-Bernstein, Mark Rosen, Susan Ryan, Sundar Sarukkai, Aparna Sharma, Tami Spector, John Stein, Anne Swartz, Tibor Tarnai, Robert Thill, Yu-Chuan Tseng, Kim Veltman, Julian Voss-Andreae, James Faure Walker, Stephan Wensveen, Louise Whiteley, Xin-She Yang, Kang Zhang, Jonathan Zilberg 